\title{
Changes in accumulation of heteroplasmic mitochondrial DNA and frequency of recombination via short repeats during plant lifetime in Phaseolus vulgaris
}

\author{
Magdalena Woloszynska ${ }^{1, \# \bowtie}$, Edyta M. Gola² and Janusz Piechota ${ }^{1}$ \\ 'Laboratory of Molecular Cell Biology, Faculty of Biotechnology; 2Department of Plant Developmental Biology, University of Wroclaw, Wrocław, \\ Poland
}

Recombination via short repeats in plant mitochondrial genomes results in sublimons - DNA molecules with a copy number much lower compared to the main mitochondrial genome. Coexistence of stoichiometrically different mitotypes, called heteroplasmy, plays an important evolutionary role, since sublimons occasionally replace the main genome resulting in a new plant phenotype. It is not clear, how frequency of recombination and sublimon production is regulated and how it is related to changes in the quantity of the main genome and sublimons. We analyzed the accumulation of two recombining main genome sequences and two resulting sublimons in apical meristems, undifferentiated tissues and leaves of different age of Phaseolus vulgaris. Copy numbers of the main genome sequences varied greatly depending on tissue type and organ age while accumulation of sublimons remained much more stable. Although the overall accumulation of plant mtDNA decreased with the leaf age, the quantity of sublimons increased relative to the main genome indicating a higher frequency of recombination via the short 314 bp repeat. Recombination was symmetrical in young developing leaves while in senescent tissues it shifted towards asymmetric events resulting in overrepresentation of one product. We propose that during plant lifetime replication and recombination frequencies change oppositely sustaining heteroplasmic compositions of the genome, which are favorable for inheritance and maintenance of complex plant mtDNA.

Key words: heteroplasmy, mtDNA recombination, Phaseolus vulgaris, plant mitochondrial genome, quantitative real-time PCR, sublimons

Received: 21 November, 2012; accepted: 28 November, 2012; available on-line: 06 December, 2012

\section{INTRODUCTION}

Mitochondrial genomes of higher plants contain recombinationally active DNA molecules varying in size, structure, stoichiometry and function (Oldenburg \& Bendich 1996; Backert et al., 1997; Manchekar et al., 2006). Recombination of plant mtDNA occurs across large (above $1 \mathrm{~kb}$ ) or short (six to several hundred base pairs) repeated sequences. Activity of short repeats results in novel substoichiometric mitotypes and gives rise to heteroplasmy - the state in which the abundant mitochondrial main genome coexists with recombinant $\mathrm{mtDNA}$ molecules at low copy number, so called sublimons (reviewed in Woloszynska, 2010). Heteroplasmy is common in plants and plays an important role in evolution of mtDNA, because sublimons can be amplified during substoichiometric shifting to replace the main genome (Janska et al., 1998). Our studies, presented here, were devoted to determining whether frequency of recombination varies in different tissues and organs during plant lifetime and how differences in recombination rate shape stoichiometry of both the main genome and sublimons. The quantity of sublimons has been estimated in different plant species as ten-, hundred- or even thousand-fold lower compared to the main genome (Arrieta-Montiel et al., 2001; Feng et al., 2009; Woloszynska \& Trojanowski, 2009). The identification of mitochondrial proteins, which suppress recombination via short repeats (Zaegel et al., 2006; Shedge et al., 2007), suggests that frequency of recombination and resulting accumulation of sublimons can be modulated during the plant lifetime and can depend on organ, tissue or cell differentiation status. Studies addressing variation in quantity of $\mathrm{mtDNA}$ are, however, limited and focused on the main mitochondrial genome, while sublimon accumulation is only sporadically investigated. An elevated amount of mtDNA in the root apical meristem (RAM) is a general phenomenon in higher plants (Kuroiwa \& Fujie, 1992; Suzuki et al., 1992; Fujie et al., 1993; Suzuki et al., 1995). Individual mitochondria contain significantly more DNA in Arabidopsis shoot apical meristem (SAM) and leaf primordia (Fujie et al., 1994) or in rice egg cells (Takanashi et al., 2010) as compared to differentiated tissues or cells, respectively. Sublimons accumulate in meristems (ArrietaMontiel et al., 2001) and undifferentiated tissues (Kanazawa et al., 1994; Albert et al., 2003). No difference in the copy number of mitochondrial DNA in Arabidopsis and tobacco was detected in young and senescent tissues $(\mathrm{Li}$ et al., 2006). On the contrary, Preuten et al. (2010) demonstrated variation in the mitochondrial genome during senescence in Arabidopsis leaves.

The mitochondrial genome of the POP line of Phaseolus vulgaris contains the short $314 \mathrm{bp}$ repeat found in four genomic environments (F1-F4) (Woloszynska et al., 2001). The F1 and F2 environments are present in the main genome while the $\mathrm{F} 3$ and $\mathrm{F} 4$ sequences are substoichiometric (Fig. 1). The F1 and F2 parental sequences constantly recombine across the short $314 \mathrm{bp}$ repeat to generate and maintain the F3 and F4 sublimons. The accumulation of parental sequences estimated in the first

e-mail: mawol@psb.vib-ugent.be

\#Present address: Magdalena Woloszynska, VIB Department of Plant Systems Biology, Ghent University, Technologiepark 927, 9052 Gent, Belgium

Abbreviations: bp, base pair; kb, kilobase; mtDNA, mitochondrial DNA; RAM, root apical meristem; SAM, shoot apical meristem. 


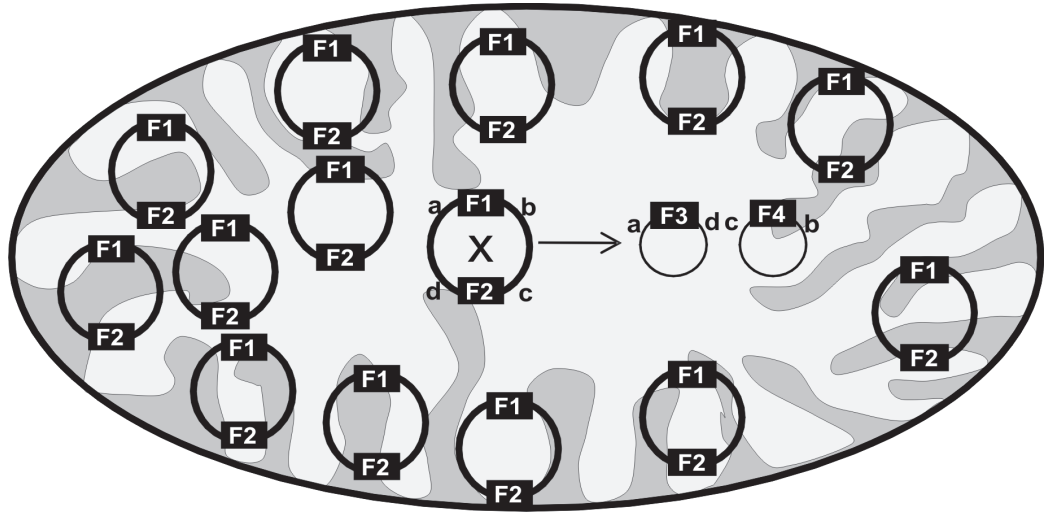

Figure 1. The short $314 \mathrm{bp}$ repeat is found in four genomic environments in the mitochondrial genome of the Phaseolus vulgaris POP line.

The main genome of the POP line (large circles) contains two copies of the short 314 bp repeat (black squares) flanked by $a$ and $b$ or $c$ and $d$ sequences in two different environments F1 and F2. Recombination between F1 and F2 across the repeated sequence generates new environments of the repeat: F3 and F4 located in sublimon molecules (small circles).

two primordial leaves of 10-day-old seedlings was found to be 100 times higher than copy numbers of substoichiometric products of recombination (Woloszynska \& Trojanowski, 2009). Presence of both substoichiometric products in balanced quantities and their constant production via recombination make the POP bean line a unique research model. In contrast, in other plants studied, only one product is usually detected or there is a vast disequilibrium between two products, and recombination across short repeats is believed to occur sporadically (reviewed in Woloszynska, 2010). Moreover, since the F1-F4 sequences interconvert via recombination,

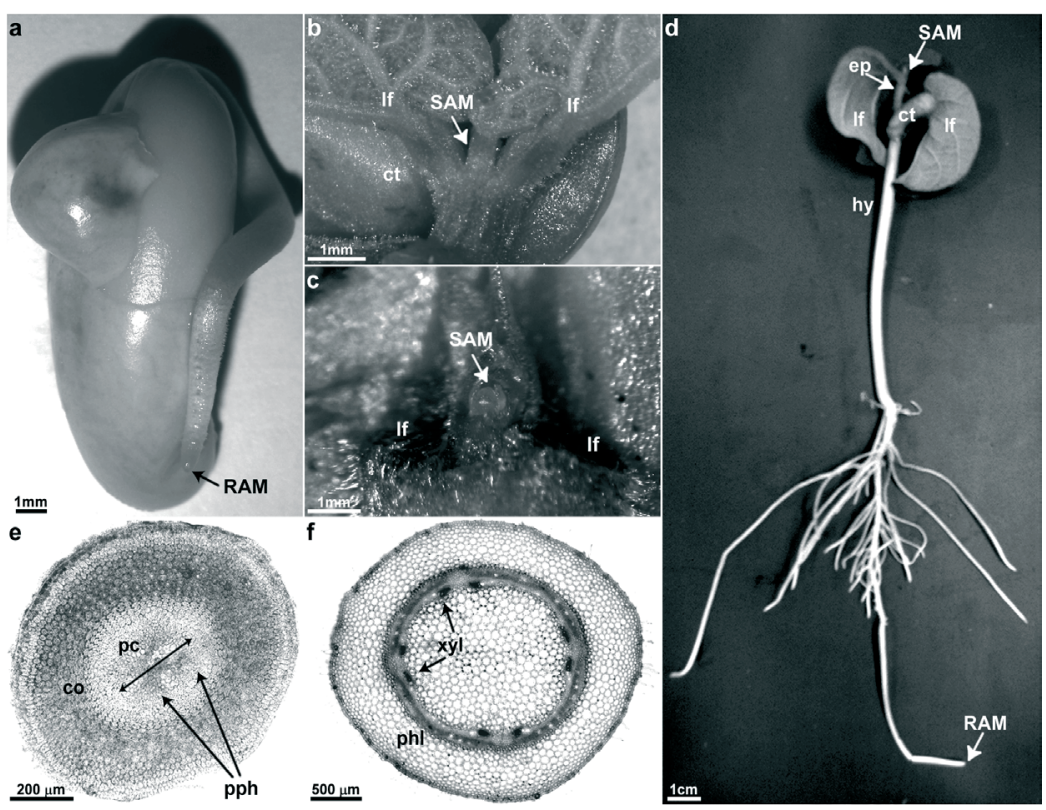

Figure 2. Plant material sampled for the mitochondrial genome analyses.

(a) an overview of the imbibed bean seed with an emerging root; the root apical meristem (RAM) is marked with an arrow. (b) an apical part of a 3-day-old seedling. One cotyledon (ct) was removed to show young developing leaves (If) and the shoot apical meristem (SAM). (c) a close-up of the SAM after dissection of leaves (If) and stipules. (d) 10-day-old seedling of common bean with cotyledons (ct), expanded leaves (If), long hypocotyl (hy), short epicotyl (ep) and well-developed root system. (e) a manualcross section of the root elongation zone. The central part of the root is occupied by meristematic cells of procambium (pc), which start differentiating to protophloem elements (pph); provascular tissue is surrounded by undifferentiated cells of the cortex (co). (f) a manual-cross section of the hypocotyl presenting differentiated phloem (phl) and xylem (xyl) tissues. changes of their relative copy number let us to conclude about variation in frequency and symmetry of recombination during plant development. We propose that the coordinated changes in accumulation and recombination of heteroplasmic plant mtDNA are tightly linked to provide a control mechanism for the accurate maintenance of mitochondrial genomes.

\section{MATERIALS AND METHODS}

Plant material and growth conditions. Common bean (Phaseolus vulgaris) seedlings were grown hydroponically at $22^{\circ} \mathrm{C}$ in a photoperiod of sixteen hours day/eight hours night. Three- or ten-day-old seedlings were used for total DNA isolation. Alternatively 10-day-old seedlings were transferred to soil and grown to obtain senescent leaves.

Material sampling. Imbibed, germinating seeds (with visible roots and cotyledons not yet released from the and the second primordial leaves, which were already an the seed, the third and the fourth leaf primeristematic tissue at the SAM, and RAMs. Three-dayold seedlings were sampled for SAMs (Fig. 2b, c), the third and the fourth leaf primordia, RAMs and the root elongation zone, containing undifferentiated and intensively growing yet non-dividing cells. Ten-day-old seedlings (Fig. 2d) were used to take the samples of SAMs, the first and the second expanded leaves, RAMs, the root elongation zone (Fig. 2e), and epicotyls (stem fragments between the first true leaves and cotyledons Fig. 2d). SAMs and leaf primordia at the meristem were sampled from seeds after dissecting of cotyledons and primordial leaves, whereas additionally in seedlings, stipules covering the apices were removed (Fig. 2b, c). RAM samples were taken from seeds as well as from seedlings (Fig. 2a, d) by cutting off the most distal part of the root, about $0.1-0.2 \mathrm{~mm}$ long. Mature fully developed green leaves and senescent leaves were collected from the same or the neighboring node.

The samples of the first and the second primordial leaves were prepared by pooling the material from 10 seeds in each sample. The samples of meristems, the third and the fourth leaf primordia, epicotyls and the root elongation zone contained the pooled plant material from 25-30 seeds or seedlings. Samples were prepared in three biological replicates.

The samples of the first and the second leaves of 10-day-old seedlings, mature green leaves and senescent leaves were obtained from individual leaves and were analyzed in at least 
ten biological replicates. All samples were analyzed at least three times by real-time PCR.

DNA isolation. We used total DNA for our analysis, because we have demonstrated previously (Woloszynska \& Trojanowski, 2009) that results of the real-time PCR quantification of the F1-F4 sequences are the same when total or mitochondrial DNA samples are used as templates for PCR. The total DNA was isolated with the Plant and Fungi DNA Purification Kit (EURx, Poland).

Quantitative real-time PCR. We used the LightCycler 2.0 instrument and the Real-Time 2×PCR Master Mix SYBR version B (A\&A Biotechnology, Poland) with optimized concentration of $\mathrm{MgCl}_{2}$. Reactions were carried out in a total volume of $10 \mu \mathrm{l}$ with a final concentration of $0.5 \mu \mathrm{M}$ primers. The primers used to amplify F1-F4 recombination forms and the cob and coxIII genes were previously described (Woloszynska \& Trojanowski, 2009). The $121 \mathrm{bp}$ fragment of the nuclear amylase gene was amplified using the AmylF (5'-ATTGACCATCTCAATCCGCAGGTGC-3') and AmylR (5'-AAATGCTAGGGGCATAGCCCT'TCAC-3') primers.

The thermal profile started with 3 min denaturation at $95^{\circ} \mathrm{C}$ followed by 40 cycles of amplification $\left(95^{\circ} \mathrm{C} / 10 \mathrm{~s}\right.$, $42^{\circ} \mathrm{C} / 5 \mathrm{~s}, 72^{\circ} \mathrm{C} / 15 \mathrm{~s}$ with single data acquisition), melting curve analysis $\left(95^{\circ} \mathrm{C} / 0 \mathrm{~min}, 65^{\circ} \mathrm{C} / 15 \mathrm{~s}, 95^{\circ} \mathrm{C} / 0 \mathrm{~s}\right.$ with slope of $0.1^{\circ} \mathrm{C} / \mathrm{s}$ and continuous data acquisition) and cooling to $40^{\circ} \mathrm{C}$ for $30 \mathrm{~s}$.

The relative copy numbers of the F1-F4 sequences as well as the cob and coxIII genes were established using the Relative Quantification option of the LightCycler $\mathrm{v}$ 4.0 software (Roche Diagnostics, Germany). DNA concentration in each sample was standardized by amplification of the reference nuclear amylase gene. The copy numbers of the F1-F4 sequences were quantified in the examined samples relative to the calibrator sample containing known accumulation of the given sequences. The arbitrary chosen sample of DNA extracted from the first two primordial leaves of 10-day-old seedlings was used as a calibrator because we have previously established that the relative copy numbers of the F1:F2:F3:F4 sequences in this biological material were 100:100:1.25:1.02, respectively (Woloszynska \& Trojanowski, 2009). The F1-F4 accumulation values presented on charts (Fig. 3a, b) for leaves of 10-day-old seedlings are averages obtained for calibrator and two other samples of DNA isolated from leaves of 10-day-old seedlings.

Statistical analysis. Two independent statistical approaches were applied to analyze various sets of sample groups. In the first approach the ANOVA analysis (Analysis of Variance) was applied to compare means from several groups of samples. Then, the HSD (Honestly Significant Difference) Tukey's post hoc test was used to find which means are significantly different from one another. The Shapiro-Wilk test was used to check the normality of sample distribution. The variance homogeneity of variances was checked using the Bartlett test. Because many times the Bartlett test indicated heterogeneity of variances, a second approach was applied in which a non-parametric Kruskall-Wallis test was performed. Then, the Student's $t$-test was applied for each pair of groups in order to check whether the differences in means are statistically significant. $P$-values from multiple comparisons with the Student's $t$-test were corrected using the Bonferroni correction method. The F (FisherSnecedore) test was applied to check the homogeneity of variances for each pair of groups. Essentially, both approaches gave the same results. MANOVA (Multivariate Analysis of Variance) was used to analyze whether differences in accumulation between the main genome se- quences F1 and F2 or between the sublimon sequences F3 and F4 were statistically significant.

\section{RESULTS}

The aim of this study was to investigate how the tissue-, organ- and age-dependent differences in accumulation of mtDNA are related to frequency of recombination across short repeat in mtDNA of the common bean. We chose meristems and undifferentiated tissues as well as leaves of different age as a biological material for our study. Meristems and undifferentiated tissues are known to be enriched in mtDNA. On the other hand, we expected that senescent leaves should contain a decreased copy number of mtDNA as was shown for plastid genomes (reviewed by Rowan \& Bendich, 2009). Moreover, proteins controlling recombination of $\mathrm{mtDNA}$ are selectively expressed in meristematic and undifferentiated tissues or in young leaves (Zaegel et al., 2006; Shedge et al., 2007) suggesting a lower recombination rate in these tissues and its higher frequency in non-dividing, differentiated and mature or even senescent tissues.

\section{Quantities of mitochondrial main genome sequences and sublimons change differently depending on tissue and organ type or age}

Apical meristems (SAM and RAM) and undifferentiated tissues (leaf primordia, stem and the root-elongation zone) were obtained from seeds and 3- and 10-dayold seedlings. To analyze the age-dependent changes in mtDNA, leaves were harvested from seeds (primordial leaves), 10-day-old seedlings (developing leaves) and from mature plants (green fully developed leaves and senescent ones). Accumulation of the F1-F4 sequences was established by the real-time PCR relative to the calibrator sample of DNA isolated from the first two developed leaves of 10-day-old-seedlings containing known copy numbers of the sequences analyzed (Materials and Methods). We used a nuclear gene as the reference raising the concern that endoreduplication of the nuclear DNA may affect the real-time PCR results. However, endoreduplication is low in leaves of the common bean and the mean DNA content per nucleus is similar in different leaves of the plant (Barrow \& Meister, 2003). Moreover, the aim of this experimental approach was to follow the differences in accumulation changes between the main genome sequences and sublimons which are not affected by putative endoreduplication divergences in the analyzed samples. As expected, the accumulation of mitochondrial sequences F1 and F2 was significantly higher in meristems and undifferentiated tissues relative to the calibrator sample (Fig. 3a). Also, accumulation of sequences F3 and F4 was usually higher in meristems and undifferentiated tissues relative to the calibrator sample (Fig. 3b). However, the increases in accumulation were not always statistically significant. In the case of the F3 sequence both applied statistical approaches indicated that accumulation in leaf primordia, SAMs of seeds and in SAMs of 3-day-old seedlings was not statistically different from the calibrator. The differences in accumulation of the F4 sequence were usually statistically insignificant. Only the amounts of the F4 sequence in RAMs of seeds (the second statistical approach), in the root elongation zone of 3-day-old seedlings (the first statistical approach), and in RAMs and the root elongation zone of 10-day-old seedlings were significantly higher compared to the calibrator. 

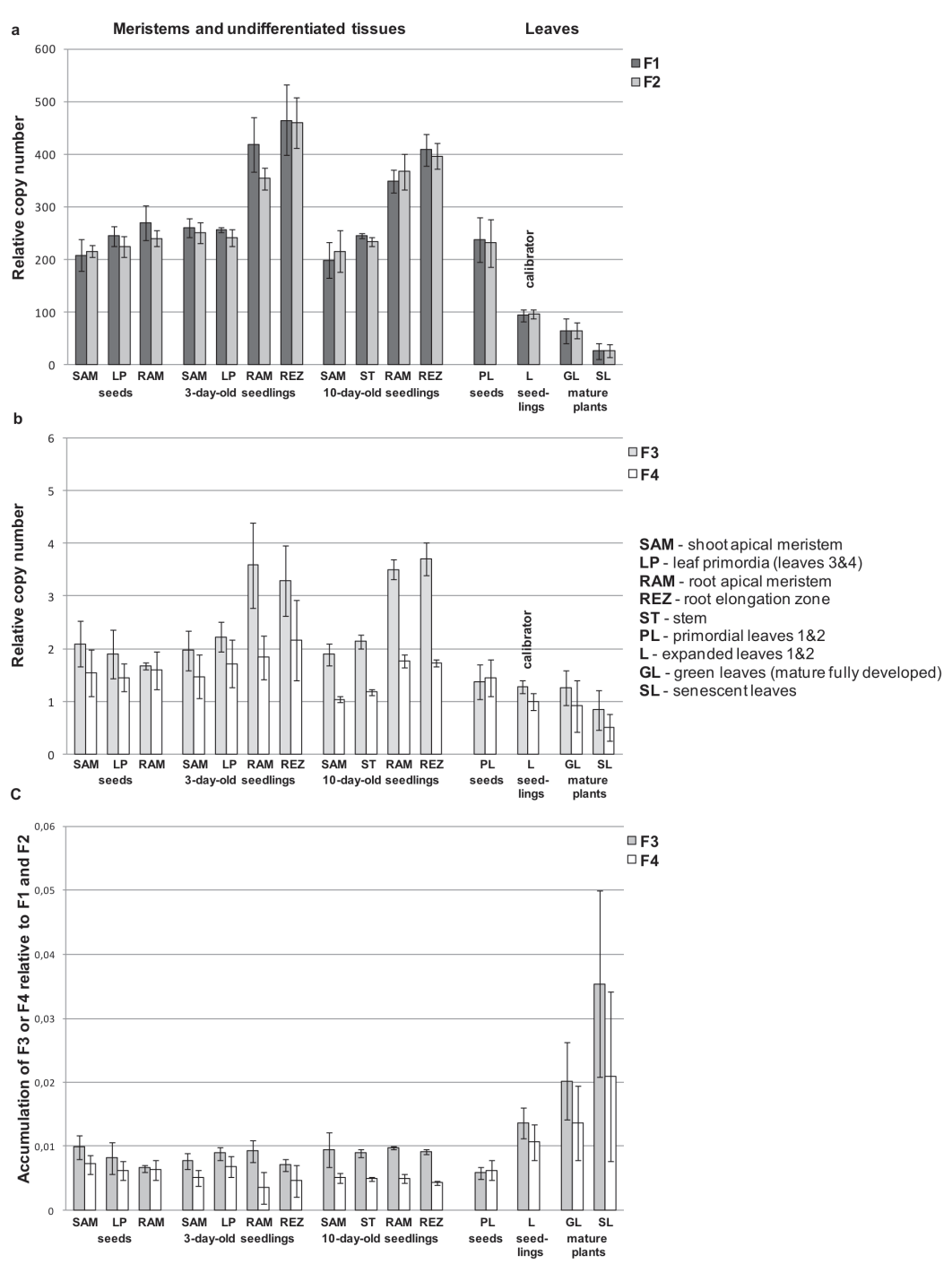

Figure 3. Age-, tissue- and organ-related accumulation of F1-F4 recombination forms. The relative copy numbers of the main genome parental recombination forms F1, F2 (a) and substoichiometric recombination products $\mathrm{F} 3, \mathrm{~F} 4$ (b) were measured by real-time PCR relative to the calibrator in leaves of different age, shoot apical meristems (SAM), leaf primordia (LP), stem (ST), root apical meristems (RAM) and a root elongation zone (REZ) isolated from seeds and 3- or 10-day-old seedlings. Since copy numbers of F1 and F2 are several hundred fold higher than copy numbers of F3 and F4, the results of the real-time PCR analysis are presented on separate charts. The accumulation of F3 or F4 sequences relative to F1 and F2 (c) was calculated in the same tissue samples and used as a measure of F1XF2 recombination frequency.

In SAMs the abundance of F1 and F2 was 2-2.5 fold higher while the quantity of F3 and F4 sublimons was maximally only 1.7 fold higher than in the calibrator. The accumulation of the mitochondrial sequences analyzed in RAMs of seeds was similar to that observed in SAMs, whereas in seedlings, RAMs contained notably higher amounts of mtDNA than SAMs (Fig. $3 a$ and b). Undifferentiated tissues derived from SAM (leaf primordia or stem) or RAM (the root-elongation zone) contained F1-F4 amounts very similar to those detected in the respective meristems (Fig. $3 \mathrm{a}$ and b). It was striking that the quantities of the main genome sequences $\mathrm{F} 1$ and F2 were very similar to each other whereas the F3 and F4 sublimons behaved differently. While F3 in general followed the pattern presented by F1 and F2 (despite the lower multiplicity of change), the profile of the F4 fluctuations was much flatter.
The content of the F1-F4 sequences in leaves was age-dependent showing the highest level in young developing leaves isolated from seeds and the lowest value in senescent leaves of ageing plants (Fig. 3a and b). The copy number of the main genome F1, F2 sequences between these two extreme developmental stages differed approximately by 7.5 -fold. On the other hand, the maximal age-related difference in the copy number of sublimon recombination products F3 and F4, observed between the youngest and the oldest leaves analyzed was only 2.5 fold. The main genome sequences $\mathrm{F} 1$ and F2 presented a very clear gradual pattern of quantitative changes: their accumulation was the highest in the primordial leaves isolated from seeds, lower in the corresponding expanded leaves of 10-day-old seedlings, again lower in the green fully developed leaves of mature plants and finally the lowest in the senescent leaves of ageing plants (Fig. 3a). The accumulation of both F1 and F2 differed significantly between all leaf age-categories (KruskalWallis test, $p$-values $=3.628 \mathrm{e}-11$ and 2.647e-16, respectively). On the contrary, the accumulation of the F3 and F4 sublimons differed to a lower extent (Kruskal-Wallis test, $p$-values $=1.835 \mathrm{e}-06$ and $9.349 \mathrm{e}-09$, respectively). In the case of F3, the differences between leaves isolated from seeds, seedlings and green leaves of mature plants (Fig. 3b) were statistically insignificant. Only senescent leaves contained significantly lower amounts of the F3 sequence. A statistically significant decrease in accumulation of the F4 sequence was observed between primordial leaves of seeds and leaves of seedlings, and between green and senescent leaves from mature plants.

Summing up, the accumulation of all four recombination forms was found to differ depending on tissue, organ-type and age but the amounts of the main genome sequences F1 and F2 (parental recombination forms) were more severely changed than the copy numbers of F3 and F4 sublimons (products of recombination).

\section{The balance between F3 and F4 recombination products is not always maintained}

In many plant species products of recombination across short mitochondrial repeats largely differ in accumulation and in some cases only one product can be detected. The F3 and F4 sequences produced by recombination via the short 314 bp repeat in the common bean mitochondrial genome were always detected together in all types of samples analyzed in these studies. However, 


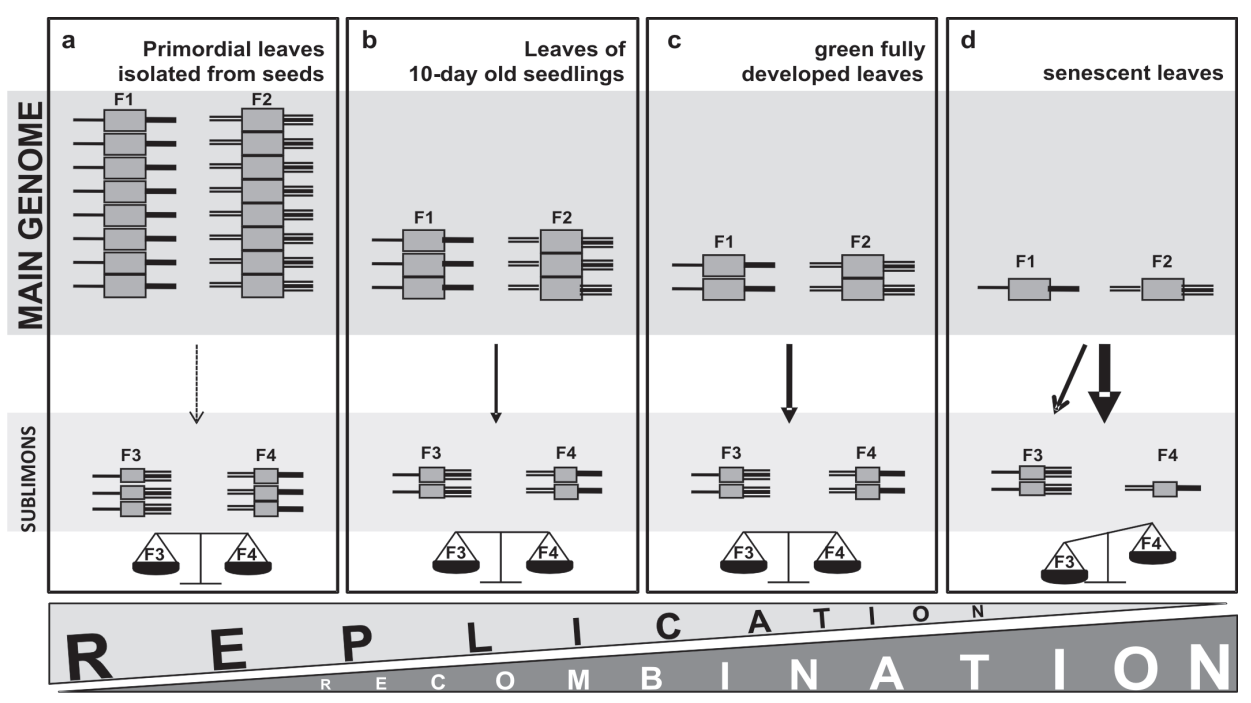

Figure 4. A model scenario integrating mtDNA recombination and replication to explain changes in heteroplasmic mtDNA, which take place during the plant lifetime.

Each recombination form has different combination of flanking sequences represented by different line styles. Different sizes of the recombination forms distinguish between main genome (large) and substoichiometric sequences (small). Diverse copy numbers of the given recombination form correspond to differences in accumulation of F1-F4 sequences during the plant lifetime. Vertical arrows varying in thickness represent the F1xF2 recombination which becomes more frequent with plant age. The diagonal arrow symbolizes possible asymmetric recombination events that may occur parallel to symmetrical recombination. Asymmetric recombination and/or preferential replication of $\mathrm{F} 3$, which lead to the unbalanced accumulation of recombination products in senescent leaves, are shown by the pan balance. relatively low in meristems and undifferentiated tissues as well as in primordial leaves isolated from seeds indicating low frequency of $\mathrm{F} 1 \times \mathrm{F} 2$ recombination in these tissues. The differences in the ratios calculated for $\mathrm{F} 3$ and F4 analyzed in all undifferentiated and meristematic tissues were statistically insignificant (Kruskal-Wallis test, $p$-value $=0.052$ and 0.11 , respectively). The gradual increase of the ratio values paralleled the leaf age suggesting that the rate of recombination across the short 314 bp repeat increases during the plant lifetime (ANOVA test, $p$-values $=1.3 \mathrm{e}-9, \quad$ and 6.1e-6, respectively). As mentioned in the previous chapter, senescent leaves, meristematic and undifferentiated tissues we found that the balanced concentrations of F3 and F4 were maintained only in seeds and in young expanded leaves of seedlings (Fig. 3b). In meristems and undifferentiated tissues of seedlings as well as in senescent leaves the copy numbers of F3 sequence were significantly higher than F4 (MANOVA test, $p$-value $=0.02$ ). Predominance of F3 was moderate in 3-day-old seedlings while during plant development it became more pronounced and in 10-day-old seedlings the F3 quantity was two-fold higher than that of F4. In senescent leaves, the F3 accumulation was about 1.7-fold higher than the abundance of F4. The amounts of the parental recombination forms F1 and F2 always remained equimolar regardless of the tissue, organ or plant age.

\section{Recombination frequency via the short repeat is low in meristems and undifferentiated tissues and increases with the leaf age}

We have previously shown that although the $314 \mathrm{bp}$ repeat belongs to the short repeated sequences believed to recombine only sporadically, the $\mathrm{F} 1 \times \mathrm{F} 2$ recombination in the POP common bean line is an active process constantly producing F3 and F4 sublimons (Woloszynska \& Trojanowski, 2009). Consequently, we assumed that more frequent $\mathrm{F} 1 \times \mathrm{F} 2$ recombination would result in increased accumulation of the substoichiometric recombination products F3 and F4 relative to accumulation of the parental F1 and F2 forms. On the contrary, low relative accumulation of $\mathrm{F} 3$ and $\mathrm{F} 4$ would point toward lower recombination rate. To follow the frequency of recombination via the 314 bp repeat, the relative accumulation of recombination products was calculated as the ratio between the copy number of F3 or F4 and the average copy number of F1 and F2. Due to unbalanced amounts of recombination products in some tissues/organs, the calculations were performed separately for F3 and F4. This ratio was used as a measure of recombination frequency (Fig. 3c). The ratio was found to be of seedlings contained unbalanced amounts of F3 and F4. Consequently, the relative accumulation of F4 was lower than F3 in these samples indicating that symmetrical recombination resulting in production of both forms was not exclusively responsible for their copy number. Apparently other mechanisms, which will be analyzed in the Discussion chapter, may shape the final stoichiometry of sublimons.

\section{Age-related changes in the F1 and F2 accumulation are representative for the main mitochondrial genome}

Since the F1 and F2 sequences recombine with frequency varying over the plant lifetime, we considered the possibility that differences in their age-related accumulation resulted from recombination and were not representative for the main mitochondrial genome as a whole. To verify this possibility, we chose two mitochondrial genes: $c o b$ and $c o x I I I$, which are present as single copies in the POP mitochondrial genome (Janska et al., 1998), are not involved in recombination events and consequently, are good candidates to represent quantitative changes of the main mitochondrial genome. We followed the relative copy number of F1, F2, cob and coxIII in the DNA samples tested earlier for F1-F4 quantity representing tissues and organs of different age. We found that changes in accumulation of $c o b$ and coxIII were very similar to those observed for F1 and F2: copy numbers of both genes were about 9 fold lower in the senescent leaves than in the SAM and about 14 fold lower than in the RAM of 10-day old seedlings. Consequently, tissue and age-related variation in the F1 and F2 copy number is representative for the mitochondrial main genome.

\section{DISCUSSION}

We have shown that during the age-related decrease of plant mtDNA quantity, accumulation of sublimons in- 
creased relative to the main genome most probably due to the higher frequency of recombination occurring via the short repeated sequence (Fig. $3 \mathrm{a}$ and b). The age-related changes of plant $\mathrm{mtDNA}$, although predictable by analogy to plastid genomes, have not earlier been clearly explained and some contradictory results have been published. Recombination across short mitochondrial repeats is often viewed as a sporadic event. Contrary to this paradigm we have not only shown that recombination of this kind may occur constantly (Woloszynska \& Trojanowski, 2009) but in this manuscript we also present data indicating that frequency of short repeat recombination can be modulated during plant development. We propose a model scenario integrating mtDNA recombination and replication to explain how quantitative changes in heteroplasmy make this state possible to maintain at the same time preventing destabilization of plant $\mathrm{mtDNA}$ (Fig. 4). Very young developing leaves contain the highest amounts of mtDNA similar to those observed in SAM and leaf primordia (Fig. 3a, b and Fig. 4). All those tissues also possess the lowest proportion of sublimons relative to the main genome (Fig. 3c). The similarity of leaf primordia and young leaves to meristems can be explained by the proliferative (meristematic) activity of their cells balanced with the cell differentiation (Poethig, 1997; Skirycz et al., 2010). This state is maintained later in young leaves during leaf-blade expansion (Donnelly et al,. 1999), where even 90\% of mesophyll cells are meristematic (Pyke et al., 1991). The intensive accumulation of mitochondrial genomes in meristems resulting from preferential replication of $\mathrm{mtDNA}$ in these tissues has already been shown by several authors (e.g. Suzuki et al. 1992, 1995). On the other hand, we found that despite the high mtDNA content in meristems the share of sublimons is low due to decreased frequency of recombination via the short 314 bp repeat (Fig. 4a). A low frequency of recombination in meristems, undifferentiated tissues and young leaves is consistent with expression patterns of known nucleus-encoded and mitochondrialocated proteins (Zaegel et al., 2006; Shedge et al., 2007), suppressing recombination, namely RecA, MutS and OSB1. Activity of these proteins provides the nuclear control acting to limit mitochondrial genome complexity by preventing recombination between small repeats. Increased replication of $\mathrm{mtDNA}$ and suppressed recombination across short repeats co-work to supply plant mitochondria with a high copy number of genomes with simplified organization. Such a mechanism facilitates the maintenance of complete genetic information during redistribution of organellar DNA in intensively dividing meristematic cells. The high copy number of sublimons in meristems relative to other tissues helps to maintain heteroplasmy during cell proliferation but at the same time, their low concentration compared to the main genome prevents substoichiometric shifting and its spread to new cells. A decrease of mtDNA copy number observed throughout the leaf development, from primordium to fully expanded mature leaf (Fig. 4a-c), is most probably a consequence of a growth-associated decrease in the replication rate related to the decline of meristematic and then undifferentiated cells. Further reduction in the accumulation of mitochondrial genomes in ageing plants (Fig. 4d) is most likely caused by DNA degradation associated with leaf senescence. Our data show that parallel to these quantitative changes, the recombination control in differentiating tissues is relaxed in an age-dependent manner. The concentration of sublimons relative to the main genome increases in young, mature and senescent leaves due to more frequent recombina- tion (Fig. 4a-d). The physiological role of narrowing of the quantitative distance between the main genome and sublimons is unclear. However, it is tempting to propose that while concentration of alternative mtDNA variants becomes closer to the main genome, they may undergo transcription and modulate mitochondrial functions during the plant lifetime. Consequently, heteroplasmy is not only the reservoir of genomic variants important in the evolutionary perspective, but it can play a significant role even within the life cycle of the individual plant.

We found that in meristematic and undifferentiated tissues, as well as in senescent leaves, the stoichiometric equilibrium between the recombination products $\mathrm{F} 3$ and F4 was lost (Fig. 3b and 4). Interestingly, in all seed samples F3 and F4 concentrations were balanced - most probably reflecting dormant mtDNA metabolism of these tissues. In all samples, where sublimon proportion was significantly unbalanced, it was always the F3 sequence which was more abundant. Interestingly, F3 (but not F4) was selectively amplified during spontaneous substoichiometric shifting, resulting in cytoplasmic male sterility in common bean (Janska et al., 1998). Unequal accumulation of recombination products with prevalence of F3 may be caused by the replicative advantage of F3 over F4 and/or asymmetric recombination. In meristems and undifferentiated tissues of seedlings mtDNA replication is very intense while recombination is suppressed as discussed earlier, thus, preferential replication seems to be the most likely mechanism responsible for higher F3 accumulation in these tissues. In maturing leaves, replication slows down, recombination accelerates and both processes achieve rates resulting in the equimolar F3/ F4 ratio. Preferential accumulation of F3 in senescent leaves can occur if symmetric recombination resulting in both F3 and F4 forms is accompanied by asymmetric recombination leading to generation of F3 alone. It is possible that asymmetric recombination may become more frequent when relaxation of the recombination control progresses during plant senescence. It should be emphasized that we have never observed a loss or dramatic decrease in F4 accumulation as it was reported for one of the products of recombination across other short repeats (reviewed in Woloszynska, 2010). Apparently, F4 is always actively maintained by recombination and the observed F3-F4 disequilibrium results from fluctuations in relative intensities of replication and symmetric/asymmetric recombination.

Taken together DNA recombination, possibly together with replication and degradation, shape differently stoichiometry of the mitochondrial main genome and sublimons during the plant lifetime. Highly stimulated replication and strictly controlled recombination of mtDNA in meristems ensure the fidelity of mtDNA inheritance and prevent substoichiometric shifting. In mature tissues, the rate of recombination increases with age giving rise to more accumulated alternative mtDNA variants. Amplification of these genome configurations during the plant lifetime can be related to recombination-dependent DNA repair found to result in increased heteroplasmy in the mitochondrial genome (Miller-Messmer et al., 2012). Amplification of substoichimetric mitochondrial DNA variants alternative to the main genome can contribute to flexibility of plant response to environmental challenges.

Our results contribute to understanding of mechanisms behind the genesis of sublimons and maintenance of complicated heteroplasmic mitochondrial genomes of higher plants. 


\section{Acknowledgments}

This work was supported by grants No. N301 015 32/0938 and NN303561039 from the Polish Ministry of Science and Higher Education.

\section{REFERENCES}

Albert B, Lelandais C, Pla M, Leuret C, Vitart V, Mathieu C, Sihachakr D, Godelle B, De Paepe R (2003) Amplification of Nicotiana sylvestris mitochondrial subgenomes is under nuclear control and is associated with phenotypic changes. Genetica 117: 17-25.

Alversone AJ, Zhuo S, Rice DW, Sloan DB, Palmer JD (2011) The mitochondrial genome of the legume Vigna radiate and the analysis of recombination across short mitochondrial repeats. PLOS ONE 6: 1-9.

Arrieta-Montiel M, Lyznik A, Woloszynska M, Janska H, Thome J, Mackenzie S (2001) Tracing evolutionary and developmental implications of mitochondrial stoichiometric shifting in the common bean. Genetics 158: 851-864.

Backert S, Nielsen BL, Borner T (1997) The mystery of the rings: structure and replication of mitochondrial genomes from higher plants. Trends Plant Sci 2: 477-483.

Barow M, Meister A (2003) Endopolyploidy in seed plamts is differently correlated to systematic, organ, life strategy and genome size. Plant Cell Environ 26: 571-584.

Donnelly PM, Bonetta D, Tsukaya H, Dengler RE, Dengler NG (1999) Cell cycling and the cell enlargement in developing leaves of Arabidopsis. Dev Biol 215: 407-419.

Feng X, Kaur AP, Mackenzie SA, Dweikat IM (2009) Substoichiometric shifting in the fertility reversion of cytoplasmic male sterile pearl millet. Theor Appl Genet 118: 1362-1370.

Fujie M, Kuroiwa H, Kawano S, Kuroiwa T (1993) Studies on the behavior of organelles and their nucleoids in the root apical meristem of Arabidopsis thaliana (L.) Col. Planta 189: 443-452.

Fujie M, Kuroiwa H, Kawano S, Muton S, Kuroiwa T (1994) Behavior of organelle and their nucleoids in the shoot apical meristem during leaf development in Arabidopsis thaliana L. Planta 194: 395-405.

Janska H, Sarria R, Woloszynska M, Arrieta-Montiel M, Mackenzie SA (1998) Stoichiometric shifts in the common bean mitochondrial genome leading to male sterility and spontaneous reversion to fertility. Plant Cell 10: 1163-1180.

Kanazawa A, Tsutsumi N, Hirai A (1994) Reversible changes in the composition of the population of $\mathrm{mtDNAs}$ during dedifferentiation and regeneration in tobacco. Genetics 138: 865-870.

Kuroiwa T, Fujie M (1992) Studies on the behavior of mitochondrial DNA. Synthesis of mitochondrial DNA occurs actively in a specific region just above the quiescent center in the root meristem of Pelargonium zonale. J Cell Sci 101: 483-493.

Li W, Ruf S, Bock R (2006) Constancy of organellar genome copy number numbers during leaf development and senescence in higher plants. Mol Gen Genet 275: 185-192.
Manchekar M, Scissum-Gun K, Song D, Khazi F, McLean SL, Nielsen BL (2006) DNA recombination activity in soybean mitochondria. $J$ Mol Biol 356: 288-299.

Miller-Messmer M, Kuhn K, Bichara M, Le Ret M, Imbault P, Gualberto JM (2012) RecA-dependent DNA repair results in increased heteroplasmy of the Arabidopsis mitochondrial genome. Plant Physiol 159: 211-226.

Oldenburg DJ, Bendich AJ (1996) Size and structure of replicating mitochondrial DNA in cultured tobacco cells. Plant Cell 8: 447-461.

Poethig RS (1997) Leaf morphogenesis in flowering plants. Plant Cell 9: 1077-1087.

Preuten T, Cincu E, Fuchs J, Zoschke R, Liere K, Borner T (2010) Fewer genes than organelles: extremely low and variable gene copy numbers in mitochondria of somatic plant cells. Plant J 64: 948-959.

Pyke KA, Marrison JL, Leech RM (1991) Temporal and spatial development of the cells of the expanding first leaf of Arabidopsis thaliana (L.) Heynh. J Exp Bot 42: 1407-1416.

Rowan BA, Bendich AJ (2009) The loss of DNA from chloroplasts as leaves mature: fact or artefact? J Exp Bot 60: 3005-3010.

Shedge V, Arrieta-Montiel M, Christensen AC, Mackenzie SA (2007) Plant mitochondrial recombination surveillance requires unusual RecA and MutS homologs. Plant Cell 19: 1251-1264.

Skirycz A, De Bodt S, Obata T, De Clerq I, Claeys H, De Rycke R, Andriankaja M, Van Aken O, Van Breusegem F, Fernie AR, Inze D (2010) Developmental stage specifity and the role of mitochondrial metabolism in the response of Arabidopsis leaves to prolonged mild osmotic stress. Plant Physiol 152: 226-244.

Suzuki T, Kawano S, Sakai A, Fujie M, Kuroiwa H, Nakamura H, Kuroiwa T (1992) Preferential mitochondrial and plastid DNA synthesis before multiple cell divisions in Nicotiana tabacum. J Cell Sci 103: 831-837.

Suzuki T, Sasaki N, Sakai A, Kawano S, Kuroiwa T (1995) Localization of organelle DNA synthesis within the root apical meristem of rice. $J$ Exp Bot 46: 19-25.

Takanashi H, Ohnishi T, Mogi M, Okamoto T, Arimura S, Tsutsumi N (2010) Studies of mitochondrial morphology and DNA amount in the rice egg cell. Curr Genet 56: 33-41.

Woloszynska M (2010) Heteroplasmy and stoichiometric complexity of plant mitochondrial genomes - though this be madness, yet there's method in't. J Exp Bot 61: 657-671.

Woloszynska M, Kieleczawa J, Ornatowska M, Wozniak M, Janska H (2001) The origin and maintenance of the small repeat in the bean mitochondrial genome. Mol Gen Genet 265: 865-872.

Woloszynska M, Trojanowski T (2009) Counting mtDNA molecules in Phaseolus vulgaris: sublimons are constantly produced by recombination via short repeats and undergo rigorous selection during substoichiometric shifting. Plant Mol Biol 70: 511-521.

Zaegel V, Guermann B, Le Ret M, Andres C, Meyer D, Erhardt M, Canaday J, Gualberto JM, Imbault P (2006) The plant-specific ssDNA binding protein OSB1 is involved in the stoichiometric transmission of mitochondrial DNA in Arabidopsis. Plant Cell 18: 3548-3563. 